University of Nebraska - Lincoln

DigitalCommons@University of Nebraska - Lincoln

July 2004

\title{
Effects of sire misidentification on estimates of genetic parameters for birth and weaning weights in Hereford cattle
}

\author{
S. L. Senneke \\ University of Nebraska-Lincoln
}

M. D. MacNeil

USDA-ARS Fort Keogh Livestock and Range Research Laboratory, Miles City, MT

L. Dale Van Vleck

University of Nebraska-Lincoln, dvan-vleck1@unl.edu

Follow this and additional works at: https://digitalcommons.unl.edu/animalscifacpub

Part of the Animal Sciences Commons

Senneke, S. L.; MacNeil, M. D.; and Van Vleck, L. Dale, "Effects of sire misidentification on estimates of genetic parameters for birth and weaning weights in Hereford cattle" (2004). Faculty Papers and Publications in Animal Science. 203.

https://digitalcommons.unl.edu/animalscifacpub/203

This Article is brought to you for free and open access by the Animal Science Department at DigitalCommons@University of Nebraska - Lincoln. It has been accepted for inclusion in Faculty Papers and Publications in Animal Science by an authorized administrator of DigitalCommons@University of Nebraska - Lincoln. 


\title{
Effects of sire misidentification on estimates of genetic parameters for birth and weaning weights in Hereford cattle ${ }^{1,2}$
}

\author{
S. L. Senneke*, M. D. MacNeil†, and L. D. Van Vleck \\ *Department of Animal Science, University of Nebraska, Lincoln 68583-0908; and \\ $\dagger$ USDA-ARS Fort Keogh Livestock and Range Research Laboratory, Miles City, MT 59301; \\ and $\ddagger$ USDA-ARS, Roman L. Hruska U.S. Meat Animal Research Center, Lincoln, NE 68583-0908
}

\begin{abstract}
Birth weights $(4,155)$ and weaning weights $(3,884)$ of Line 1 Herefords collected at the Fort Keogh Livestock and Range Research Laboratory in Miles City, MT, between the years of 1935 to 1989 were available. To study the effect of misidentification on estimates of genetic parameters, the sire identification of calf was randomly replaced by the identification of another sire based on the fraction of progeny each sire contributed to a yearly calf crop. Misidentification rates ranged from 5 to $50 \%$ with increments of $5 \%$. For each rate of misidentification, 100 replicates were obtained and analyzed with single-trait and two-trait analyses with a restricted maximum likelihood (REML) algorithm. Two different models were used. Both models contained year $\times$ sex combinations and ages of dam as fixed effects, calendar birth date as a fixed covariate, and random animal and maternal genetic effects and maternal permanent environment effects. Model 2 also included sire $\times$ year combinations as random effects. As the rate of misidentification increased, estimates of the
\end{abstract}

direct-maternal genetic correlation increased for both traits, with both models, for all analyses. With singletrait analyses, estimates of the fraction of variance that were due to sire $\times$ year interaction effects increased slightly for birth weight (near zero) and decreased slightly $(0.015$ to 0.004$)$ for weaning weight as misidentification increased. With two-trait analyses, estimates of fraction of variance that were due to sire $\times$ year effects gradually decreased for weaning weight as misidentification increased. With the two-trait analyses, and with both models, as the level of sire misidentification increased, estimates of the genetic correlation between direct effects gradually increased, and estimates of the correlation between maternal effects gradually decreased. Estimates of the direct-maternal genetic correlation were more positive with Model 2 than with Model 1 for all levels of misidentification. Results of this study indicate that misidentification of sires would severely bias estimates of genetic parameters and would reduce genetic gain from selection.

Key Words: Cattle, Genetic Correlations, Heritability

(c)2004 American Society of Animal Science. All rights reserved.

J. Anim. Sci. 2004. 82:2307-2312

\section{Introduction}

Misidentification of sires may be an important weakness in selection programs in the cattle industry. Sire misidentification causes the estimate of a negative direct-maternal genetic correlation to become positive (Lee

\footnotetext{
${ }^{1}$ Published as paper No. 14204, Journal Ser., Nebraska Agric. Res. Div., Univ. of Nebraska, Lincoln 68583-0908. A portion of this research was conducted under a cooperative agreement between USDA-ARS and the Montana Agric. Exp. Stn.

${ }^{2}$ Mention of a proprietary product does not constitute a guarantee or warranty of the product by USDA, Montana Agric. Exp. Stn., or the authors and does not imply its approval to the exclusion of other products that may also be suitable.

Correspondence-phone: 402-472-6010; fax: 402-472-6362; e-mail: lvanvleck@unlnotes.unl.edu.

Received August 1, 2003.

Accepted May 12, 2004.
}

and Pollak, 1997a), decreases estimates of both direct and maternal heritability (Lee and Pollak, 1997a), decreases genetic gain (Geldermann et al., 1986; Israel and Weller, 2000), and biases evaluations of sires (Van Vleck, 1970) and estimates of breeding values (Banos et al., 2001). Birth weight and weaning weight are economically important traits. Breeders want breeding systems that will maximize profit, which can be done by genetically improving economic traits. A common animal model used for genetic evaluation of beef cattle includes a fixed covariate of calendar birth date, fixed effects of year of birth, sex of calf, age of dam, and their interactions; random direct and maternal genetic effects; and uncorrelated permanent environmental effect of the dam. Inclusion of a sire $\times$ year interaction effect in the model has been shown to decrease bias in the estimate of the directmaternal genetic correlation (Lee and Pollak, 1997b). Therefore, a specific question to answer is whether a 
model with or without sire $\times$ year interaction effects would more accurately estimate genetic parameters from data with some misidentification of sires. The purposes of this study were to determine the effects of sire misidentification on estimates of genetic parameters, direct-maternal genetic correlations within a trait and direct genetic and maternal genetic correlations between traits, and to determine whether a model with sire $\times$ year interaction effects would more accurately estimate genetic parameters than a model without sire $\times$ year interaction effects.

\section{Materials and Methods}

\section{Data}

Records from Line 1 Herefords collected from 1935 through 1989 at the Fort Keogh Livestock and Range Research Laboratory (LARRL) located in Miles City, MT, were available on a total of 4,291 animals. The 4,291 animals had 4,155 records for birth weight and 3,884 records for weaning weight. Fort Keogh is approximately $671 \mathrm{~m}$ above sea level. Average annual precipitation is $34 \mathrm{~cm}$, of which $21 \mathrm{~cm}$ occurs from March to July. Temperatures average $-9^{\circ} \mathrm{C}$ in January and $23^{\circ} \mathrm{C}$ in July (MacNeil et al., 1992). Development of inbred lines, at LARRL, began in 1934 with the first calves in Line 1 (Knapp et al., 1951). The foundation sires of Line 1 were Advance Domino 20th and Advance Domino 54th, which were sons of Advance Domino 13th (MacNeil et al., 1992). Management of Line 1 was fairly constant from 1935 to 1989. Most calves were weighed within $24 \mathrm{~h}$ after birth. Those that survived were weighed at weaning at approximately $180 \mathrm{~d}$ (MacNeil et al., 1992).

\section{Random Misidentification of Sires}

Identification of sires was randomly assigned incorrectly at rates of $5,10,15,20,25,30,35,40,45$, and $50 \%$ for each calf crop. The incorrect identification assigned was that of another bull producing calves that same year. Assignment of incorrect identification of the sire was random and proportional to the number of progeny the other sires had each year. New starting values for the sequence of random numbers were used for each replicate so that progeny had an equal chance of becoming misidentified for each replicate. The data sets with misidentified sires were then analyzed using a derivative-free algorithm to obtain restricted maximum likelihood (REML) estimates of variance and covariance components. Averages of estimates for 100 replicates were obtained for each rate of sire misidentification. For each rate of misidentification, averages were then compared with estimates of genetic parameters from the original data, which had no deliberate misidentification.

\section{Models}

Genetic parameters were estimated with two different models for both univariate and bivariate analyses. Model

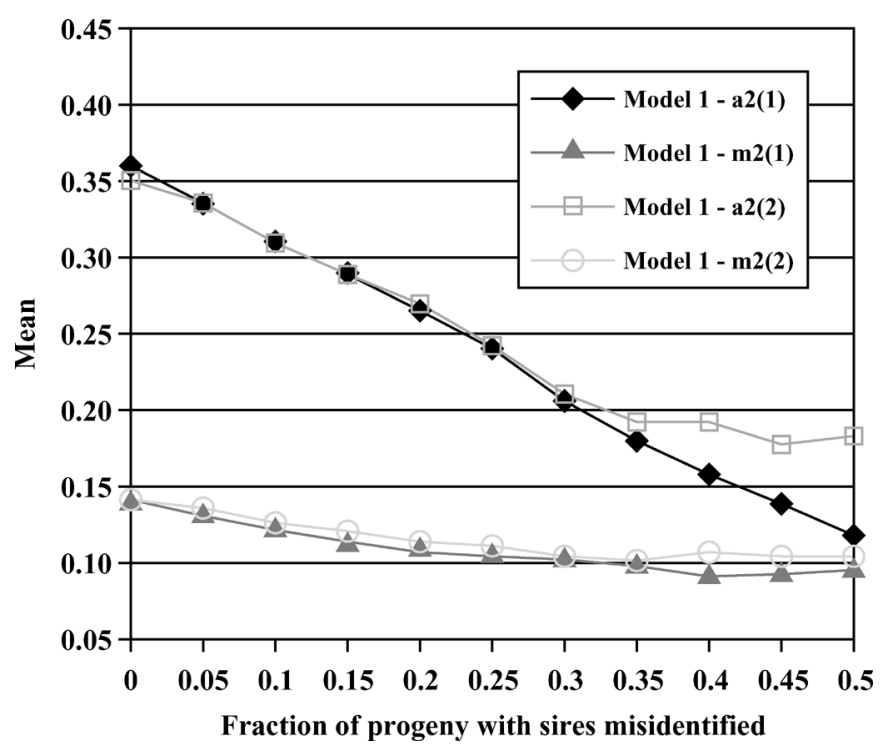

Figure 1. Means of 100 estimates of heritability and maternal heritability for each fraction of progeny with sires misidentified with Model 1 for birth weight from single-trait and two-trait analyses. Heritability from single-trait analyses $=\mathrm{a} 2(1)$; heritability from two-trait analyses $=\mathrm{a} 2(2)$; maternal heritability from single-trait analyses $=\mathrm{m} 2(1)$; maternal heritability from two-trait analyses $=\mathrm{m} 2(2)$. Model 2 is not shown because estimates were the same for Models 1 and 2 .

1 included a linear covariate of calendar birth date, fixed effects of year of birth $\times$ sex of calf and of age of dam, and random animal genetic, maternal genetic, and maternal permanent environment effects in addition to residual effects. Model 2 also included sire $\times$ year interaction as another random factor.

\section{Estimation of Variance Components}

For each replicate of data with misidentified sires, variance components were estimated with Models 1 and 2 with the MTDFREML program (Boldman et al., 1995). For univariate analyses, the stopping criterion was set at $1 \times 10^{-6}$ for the variance of the $-2 \log \mathrm{L}$ in the simplex. The number of rounds allowed per start was 200. A total of one start and three restarts was done for each replicate. Analyses for most of the replicates converged after the first restart. For the bivariate analyses, the stopping criterion was also set at $1 \times 10^{-6}$. After a few preliminary analyses, the number of rounds allowed for each start (or restart) for the bivariate analyses was set to 600 because many bivariate analyses were not converging by 200 rounds. One start and four restarts were allowed for each replicate. After the one start and four restarts allowed for each replicate, approximately $4 \%$ of the analyses had not converged. For the replicates that had not converged, the ending values from their last restart were used to restart with six additional re- 


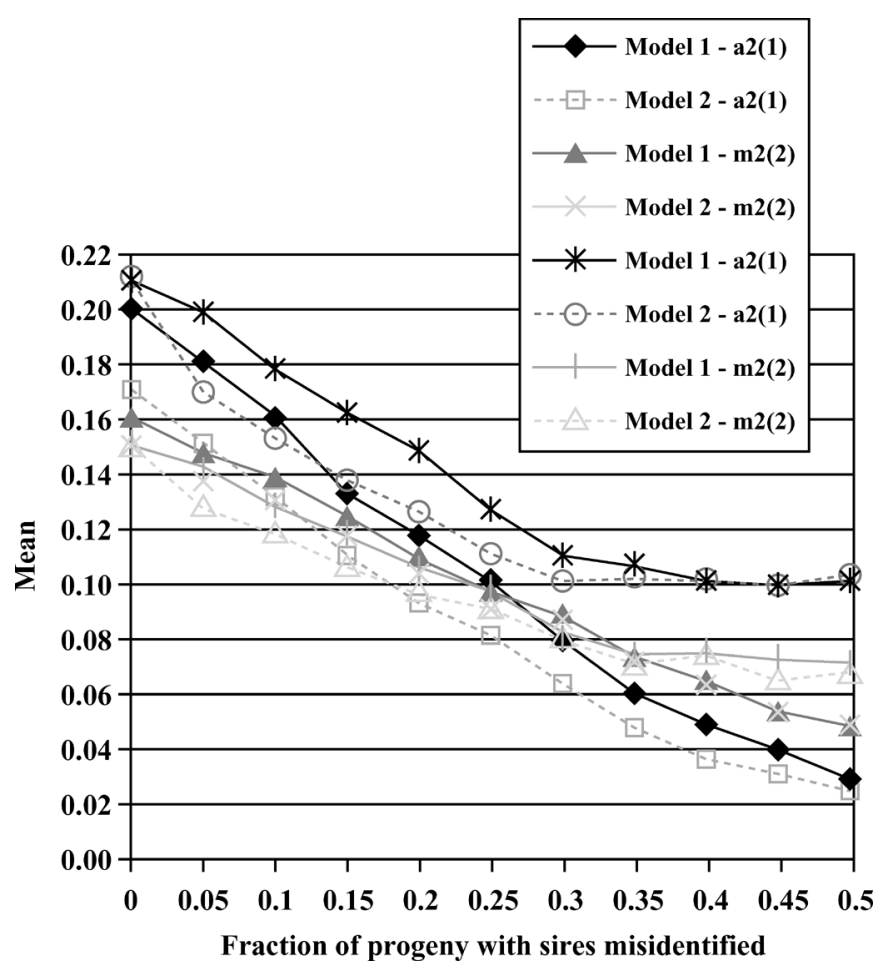

Figure 2. Means of 100 estimates of heritability and maternal heritability for each fraction of progeny with sires misidentified with Model 1 and Model 2 for weaning weight from single-trait and two-trait analyses. Heritability from single-trait analyses = a2(1); heritability from two-trait analyses = a2(2); maternal heritability from single-trait analyses $=\mathrm{m} 2(1)$; maternal heritability from twotrait analyses $=\mathrm{m} 2(2)$.

starts to ensure convergence. For the single replicate that still had not converged, an additional start and six restarts resulted in convergence. For the bivariate analyses that used Model 2, the sire $\times$ year component of variance was held constant at 0.10 for birth weight, which was approximately $0.6 \%$ of the total phenotypic variance because during preliminary analyses, some analyses would converge after one restart and then not converge at all after another restart. The sire $\times$ year variance for birth weight was a small amount and the estimate would continue to decrease toward zero. Because this estimate continued to change, the log likelihood would keep changing, so that convergence would never be met. After this variance was set to 0.10 , if the analysis of a replicate reached local convergence once, then the subsequent restarts would reach local convergence again, and finally global convergence. Estimates from each of the 100 replicates for each misidentification rate and trait were then averaged and empirical standard deviations were calculated.

\section{Results and Discussion}

As shown in Figure 1 for birth weight, for all analyses, as the fraction of misidentified progeny increased, aver-

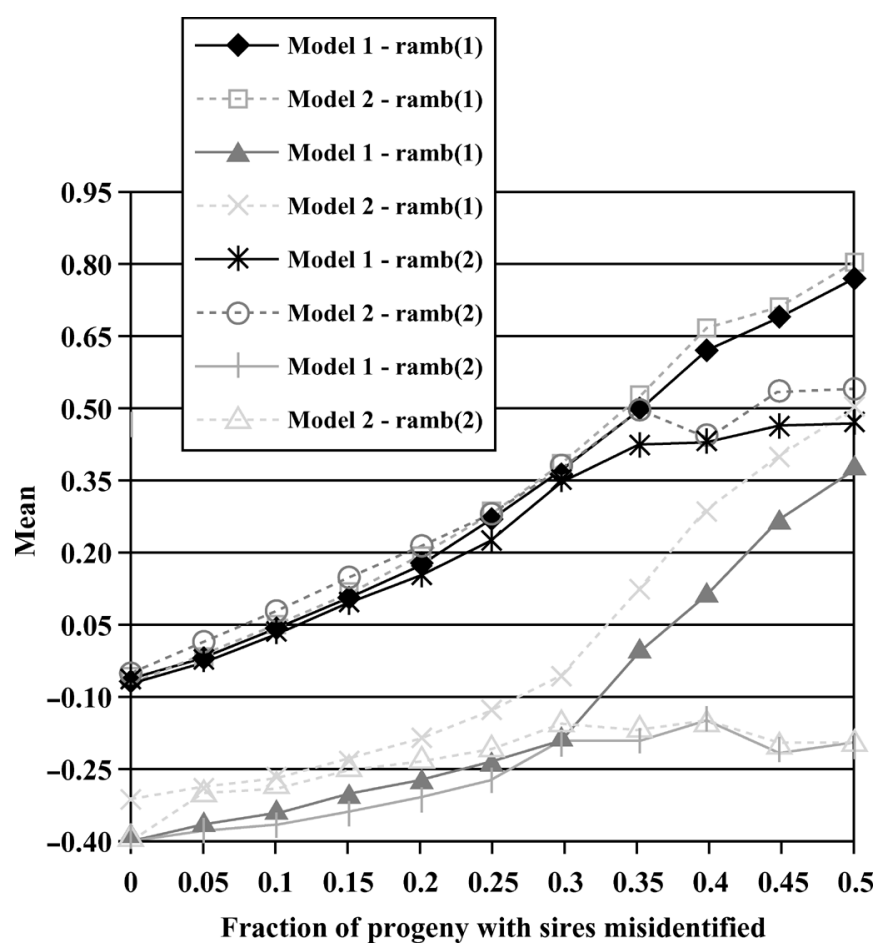

Figure 3. Means of 100 estimates of direct-maternal correlation for each fraction of progeny with sires misidentified with Models 1 and 2 for birth and weaning weights from single and two-trait analyses. Direct-maternal correlation for birth weight from single-trait analyses = ramb(1); direct-maternal correlation for birth weight from two-trait analyses $=\mathrm{ramb}(2)$; direct-maternal correlation for weaning weight from single-trait analyses = ramw(1); direct-maternal correlation for weaning weight from two-trait analyses $=\operatorname{ramw}(2)$.

age estimates of direct and maternal heritability decreased almost linearly, although the decrease in maternal heritability was not as great as the decrease in direct heritability. Estimates of both additive and maternal variance decreased dramatically, whereas the estimate of the phenotypic variance remained relatively unchanged as misidentification increased, which resulted in the decreased estimates of both direct and maternal heritability. Estimates of the phenotypic variance would not be expected to change because the same records are analyzed. With single-trait analyses, estimates of direct heritability decreased more than with two-trait analyses. Estimates of both direct and maternal heritability were the same for Models 1 and 2 .

For weaning weight, average estimates of direct and maternal heritability decreased linearly as the fraction of misidentification increased, for all analyses, although, unlike birth weight, inclusion of sire $\times$ year effects (Model 2 ) affected estimates of genetic parameters (Figure 2). With Model 2, average estimates of direct and maternal heritabilities were less than with Model 1. Robinson (1994) and Lee and Pollak (1997b), with simulated data, found that estimates of genetic parameters were less with sire $\times$ year interaction effects in the model. 
Figure 3 shows that with single-trait analyses, average estimates of the direct-maternal genetic correlation increased greatly as the fraction of misidentified progeny increased; from -0.06 to 0.78 and -0.38 to 0.38 , for 0 and $50 \%$ misidentification, for birth weight and weaning weight, respectively. Lee and Pollak (1997b) also reported that sire misidentification caused the estimate of the direct-maternal genetic covariance to be more positive than when data were simulated without misidentification. The increase in the direct-maternal correlation with increased misidentification was due to both an increase in the direct-maternal genetic covariance and decreases in the direct and maternal genetic variances. A similar pattern was found with two-trait analyses, although the increase in the average estimate of the direct-maternal genetic correlation was not as great as with single-trait analyses. The increase in the estimate of the direct-maternal genetic correlation for weaning weight with Model 2 was greater than with Model 1 $(-0.31$ to 0.50 for 0 and $50 \%$ misidentification, respectively), which is consistent with a previous study (Lee and Pollak, 1997a), although there was no difference between the two models for birth weight. Meyer (1997) reported that including sire $\times$ year interaction effects in the model decreased estimates of direct and maternal heritability and increased the estimate of the directmaternal genetic correlation for weaning weight in polled Herefords. For both birth and weaning weights, the empirical standard deviations of the direct-maternal genetic correlation increased markedly as misidentification increased. With single-trait analyses, empirical standard deviations increased from 0.04 to 0.18 and 0.03 to 0.54 for birth and weaning weights, respectively, and with two-trait analyses, empirical standard deviations increased from 0.05 to 0.30 and 0.03 to 0.28 for birth and weaning weights.

As the fraction of misidentified progeny increased, the proportion of variance that was due to maternal permanent environmental effects increased only slightly for both birth and weaning weights (Figure 4). The slight increase would be expected to compensate for the phenotypic variance remaining unchanged and decreases in the direct and maternal genetic variances. Lee and Pollak (1997a) reported that misidentification caused an increase in the estimate of the proportion of variance due to maternal permanent environmental effects for weaning weight.

For birth weight, Figure 5 shows that average estimates of the sire $\times$ year variance component with Model 2 increased slightly as the rate of misidentification increased but were a negligible fraction of the phenotypic variance. For weaning weight, the estimates, even though small, decreased as the fraction of misidentification increased (from $1.5 \%$ of the phenotypic variance with no misidentification to $0.4 \%$ at $50 \%$ misidentification), which was in contrast to birth weight, for which there was little difference in estimates of the proportion of variance due to sire $\times$ year interaction effects for different rates of misidentification.

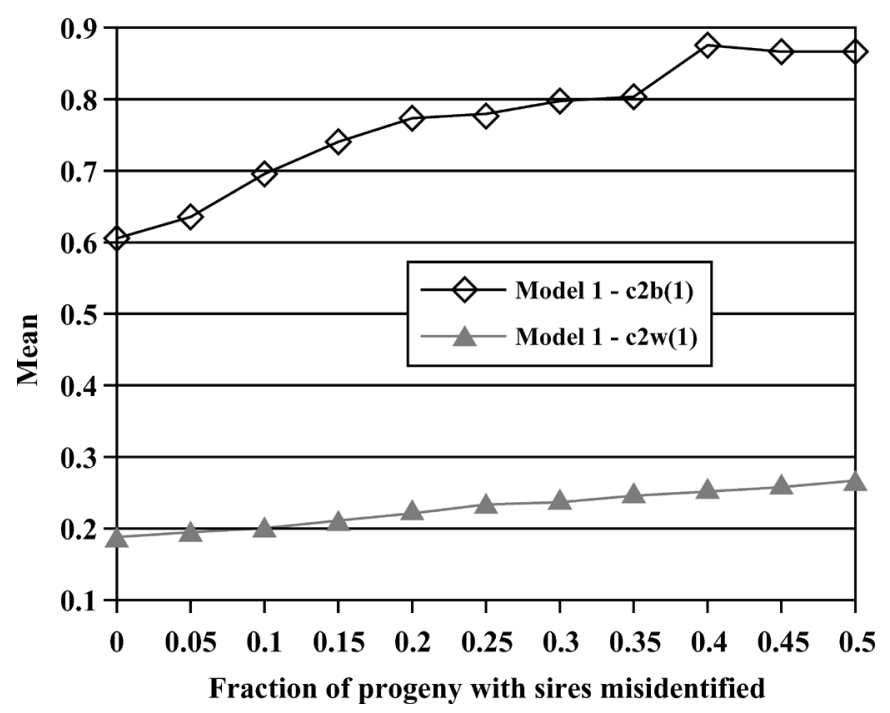

Figure 4. Means of 100 estimates of proportion of variance due to maternal permanent environmental effects with Model 1 for birth weight and weaning weight from single-trait analyses. Proportion of variance due to maternal permanent environmental effects for birth weight $=$ $\mathrm{c} 2 \mathrm{~b}(1)$; Proportion of variance due to maternal permanent environmental effects for weaning weight $=c 2 w(1)$. Model 2 and two-trait analyses are not shown because estimates were the same for Models 1 and 2, for all analyses.

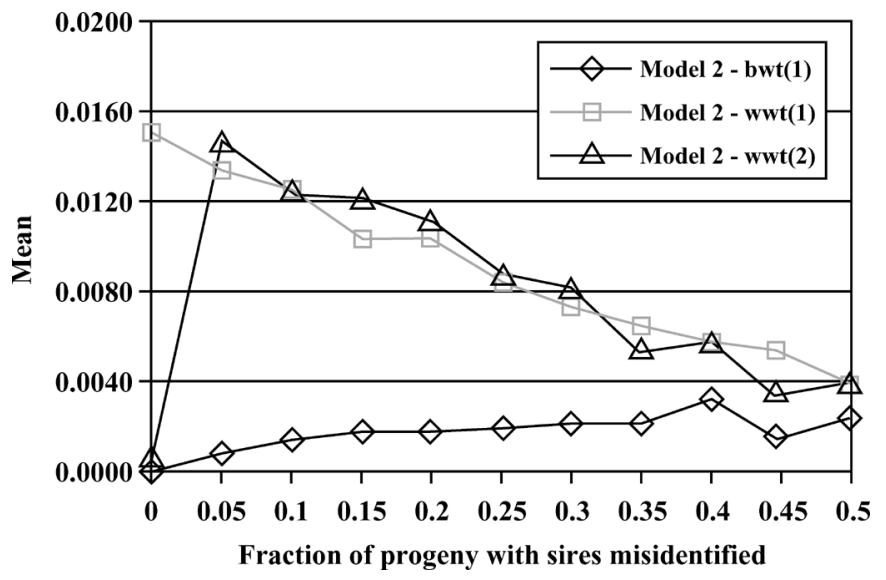

Figure 5. Means of 100 estimates of sire $\times$ year proportion of variance for each fraction of progeny with sires misidentified for birth weight and weaning weight from single-trait and two-trait analyses. Sire $\times$ year proportion of variance for birth weight $=b w t$; sire $\times$ year proportion of variance for weaning weight $=w w t$; sire $\times$ year propor tion of variance from two-trait analyses for weaning weight $=$ wwt2. Sire $\times$ year proportion of variance for birth weight is not shown for two-trait analyses because the sire $\times$ year variance was fixed at 0.10 , which was about $0.6 \%$ of the total phenotypic variance. 


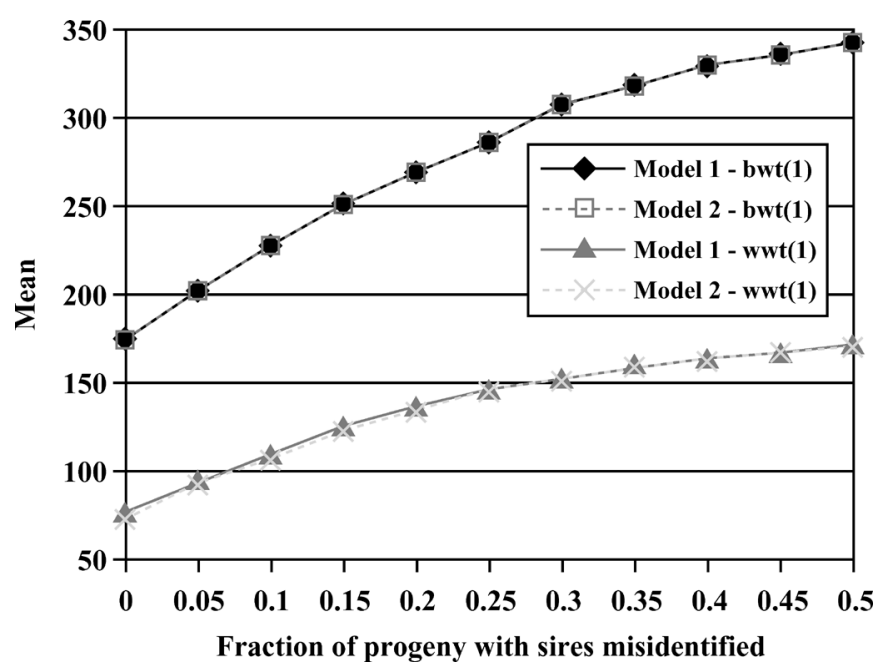

Figure 6. Means of 100 replicates of $-2 \log$ likelihood (excluding the common first two digits) for each fraction of progeny with sires misidentified with Models 1 and 2 for birth and weaning weights from single-trait analyses.

Figure 6 shows averages of -2logL for both models for birth and weaning weights. A smaller value of $-2 \log \mathrm{L}$ would indicate a better fit of the model. The observation that inclusion of sire $\times$ year effects in the model did not affect estimates of genetic parameters for birth weight is supported by the lack of a difference in the -2logL for Models 1 and 2. As misidentification increased, $-2 \log \mathrm{L}$ increased for both birth weight and weaning weight, which shows that the model fit the data better with less misidentification.

The main objective was to determine the impact of sire misidentification on the estimate of the genetic correlation between direct genetic effects for birth weight and weaning weight. Figure 7 shows that with Model 1, as the rate of misidentification increased from 0 to $40 \%$, average estimates of the direct genetic correlation between birth weight and weaning weight gradually increased, from 0.63 to 0.77 , but after $40 \%$ misidentification, average estimates decreased slightly. Average estimates of the correlation between maternal genetic values for birth and weaning weights decreased slightly from 0 to approximately $25 \%$ misidentification with a greater decrease from 25 to $50 \%$ misidentification. Estimates with Model 2 of the correlation between direct genetic values for birth and weaning weights increased markedly from 0 to $5 \%$ misidentification (0.66 to 0.77 ), but then essentially did not change from 20 to $50 \%$ misidentification. Estimates of the direct genetic correlation were greater with Model 2 than with Model 1, although the difference may have little practical importance. Average estimates of the correlation between maternal genetic effects for birth and weaning weight tended to decrease as misidentification increased. Empirical standard deviations increased greatly as misidentification increased for both estimates of the direct genetic correlation (0.04 to 0.17 and 0.05 to 0.15 for Models 1 and 2, respectively), and of the maternal genetic correlation (0.06 to 0.25 and 0.06 to 0.22 for Models 1 and 2, respectively) between the two traits. Average estimates of the

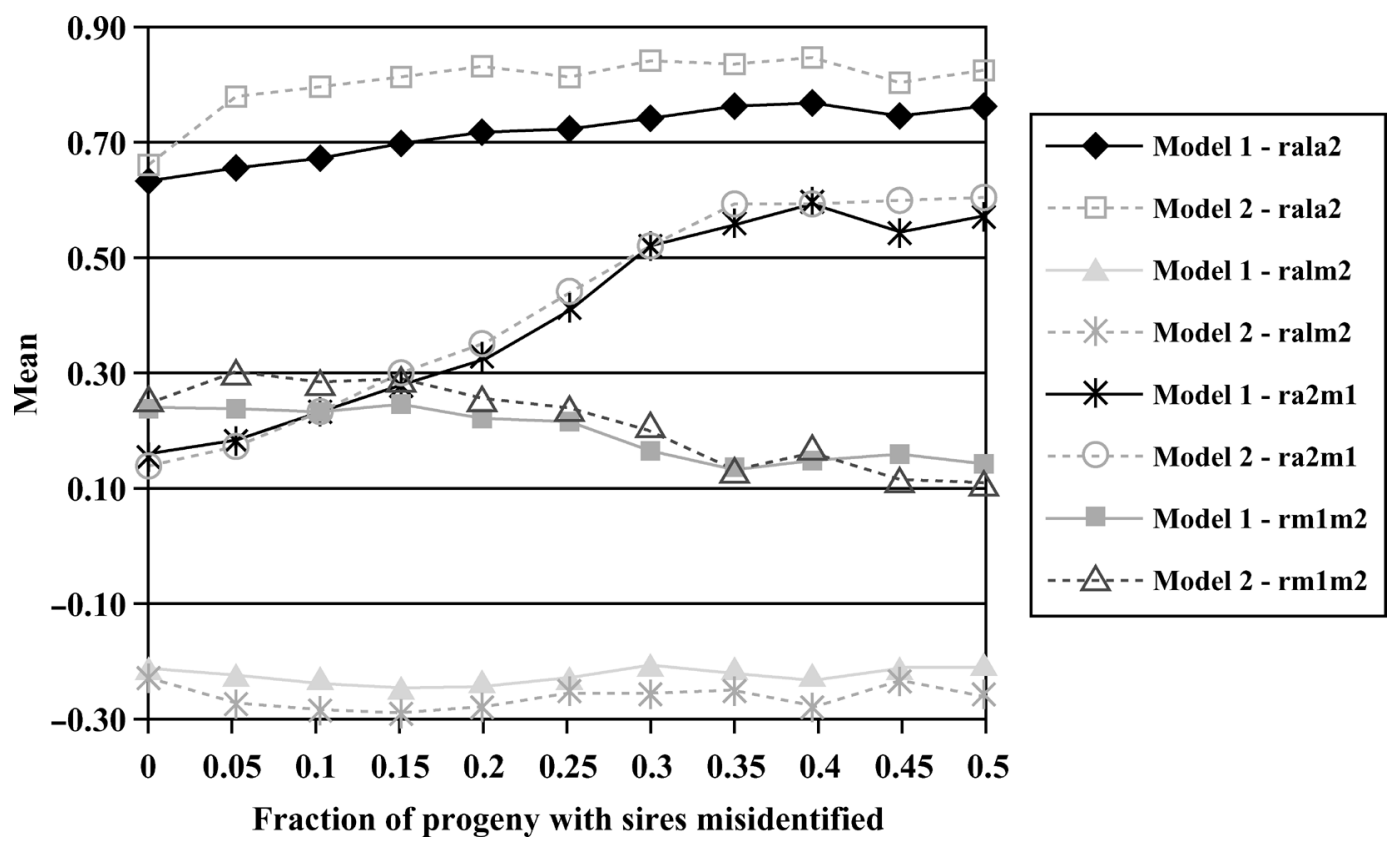

Figure 7. Means of 100 estimates of correlations between birth weight and weaning weight for each fraction of progeny with sires misidentified with Model 1 and Model 2. Genetic correlation between direct effects for birth weight and weaning weight $=$ ra1a2; genetic correlation between direct genetic birth weight and maternal genetic weaning weight $=$ ra1m2; genetic correlation between direct genetic weaning weight and maternal genetic birth weight $=$ ra2m1; genetic correlation between maternal effects for birth weight and weaning weight $=\mathrm{rm} 1 \mathrm{~m} 2$. 
genetic correlation between direct genetic value for birth weight and maternal genetic value for weaning weight changed little with either Model 1 or Model 2 as the fraction of misidentified sires increased although the empirical sampling standard deviations of the correlation increased from 0.03 to 0.24 and 0.04 to 0.24 for $5 \%$ vs. $50 \%$ misidentification for Models 1 and 2, respectively. The averages of the correlation between direct genetic value for weaning weight and maternal genetic value for birth weight increased greatly as misidentification of sires increased with both models from approximately 0.15 with no deliberate misidentification to approximately 0.60 with $50 \%$ misidentification. Most of the increase was in place by approximately $35 \%$ misidentification. Empirical sampling standard deviations increased from 0.05 to 0.32 and 0.06 to 0.27 for $5 \%$ vs. $50 \%$ misidentification for Models 1 and 2, respectively.

General Conclusions. Sire misidentification is expected to decrease the range of estimated breeding values (Geldermann et al, 1986), which is again supported by the results of this study. A decrease in the estimate of the additive genetic variance would cause a decrease in the range of estimated breeding values, whereas the estimate of the phenotypic variance remains unchanged, and hence would decrease the estimate of heritability. Although misidentification of sires had a large effect on estimates of both direct and maternal heritabilities, with a larger decrease in estimates of direct heritability, the largest effect was on the estimate of the direct-maternal genetic correlation within each trait, with an increase in the estimates of the direct-maternal genetic correlation as misidentification increased. The increases and decreases in estimates of parameters were generally linear with levels of misidentification. Therefore, rather than attempting to determine an amount of misidentification that might not be important, the best policy would be to increase correct identification as much as possible. The inclusion of sire $\times$ year interaction effects affected estimates of genetic parameters for weaning weight, in contrast to birth weight for which estimates from Models 1 and 2 were the same. Overall, estimates of the proportion of variance due to sire $\times$ year interaction effects were small and did not change much with misidentification, but for weaning weight, as the proportion of variance due to sire $\times$ year interaction effects decreased slightly, the estimate of the direct-maternal genetic correlation increased greatly. The estimate of the correlation between direct genetic effects for birth and weaning weights increased as the rate of misidentification increased, whereas the estimate of the correlation between maternal genetic effects decreased, although the magnitude of the changes may be of little practical importance. Data were from an inbred line, so the range of estimated breeding values might be less than would be expected from an outbred population, owing to an inbred population being more closely related and thus being more similar in estimated breeding values. Therefore, the consequences of misidentification of sires on estimates of genetic parameters in this study may be less severe than in an outbred population.

\section{Implications}

Misidentification of sires can bias estimates of parameters needed for national genetic evaluations, such as direct and maternal heritabilities and the direct-maternal genetic correlation. The biases will increase as misidentification increases. One consequence of misidentification would be decreased genetic gain from selection with a corresponding potential loss of income; therefore, misidentification should be decreased as much as possible. One method to increase correct identification would be to DNA-test all cattle in a herd, but the increase in genetic gain and income to producers may not be cost effective owing to the cost associated with the testing. A cost-benefit analysis together with a simulation study of long-term selection would be necessary to determine the marginal cost of identification testing. More intensive management during breeding and calving seasons might be cost effective.

\section{Literature Cited}

Banos, G., G. R. Wiggans, and R. L. Powell. 2001. Impact of paternity errors in cow identification on genetic evaluations and international comparisons. J. Dairy Sci 84(11):2523-2529.

Boldman, K. G., L. A. Kriese, L. D. Van Vleck, C. P. Van Tassell, and S. D. Kachman. 1995. A manual for use of MTDFREML. A set of programs to obtain estimates of variance and covariances [Draft]. USDA-ARS, Clay Center, NE.

Geldermann, H., U. Pieper, and W. E. Weber. 1986. Effect of misidentification on the estimation of breeding value and heritability in cattle. J. Anim. Sci. 63:1759-1768.

Israel, C., and J. I. Weller. 2000. Effect of misidentification on genetic gain and estimation of breeding value in dairy cattle populations. J. Dairy Sci. 83:181-187.

Knapp, B., R. C. Church, and A. E. Flower. 1951. Genetic History of Line 1 Hereford Cattle at the United States Range Livestock Experiment Station. Miles City, Montana. Bull. 479. Montana State College, Agric. Exp. Stn., Bozeman.

Lee, C., and E. J. Pollak. 1997a. Influence of sire misidentification on sire $\times$ year interaction variance and direct-maternal genetic covariance for weaning weight in beef cattle. J. Anim. Sci. 75:2858-2863.

Lee, C., and E. J. Pollak. 1997b. Relationship between sire $\times$ year interactions and direct-maternal genetic correlation for weaning weight of Simmental cattle. J. Anim. Sci. 75:68-75.

MacNeil, M. D., J. J. Urick, S. Newman, and B. W. Knapp. 1992. Selection for postweaning growth in inbred Hereford cattle: The Fort Keogh, Montana Line 1 example. J. Anim. Sci. 70:723-733.

Meyer, K. 1997. Estimates of genetic parameters for weaning weight of beef cattle accounting for direct-maternal environmental covariances. Livest. Prod. Sci. 52:187-199.

Robinson, D. L. 1994. Models which might explain negative correlations between direct and maternal genetic effects. Proc. 5th World Congr. Genet. Appl. Livest. Prod., Guelph. 18:378.

Van Vleck, L. D. 1970. Misidentification and sire evaluation. J. Dairy Sci. 53:1697-1702. 